\title{
Relativistic effects in EELS of nanoporous alumina membranes
}

\author{
N. Zabala, ${ }^{1,2}$ A. G. Pattantyus-Abraham, ${ }^{3}$ A. Rivacoba, ${ }^{2,4}$ F. J. García de Abajo, ${ }^{2}$ and M. O. Wolf ${ }^{3}$ \\ ${ }^{1}$ Elektrika eta Elektronika Saila, Zientzia Fakultatea, UPV/EHU, 644 Posta Kutxa, 48080 Bilbao, Spain \\ ${ }^{2}$ Donostia International Physics Center (DIPC) and Centro Mixto CSIC-UPV/EHU, Apartado 1072, 20080 Donostia, Spain \\ ${ }^{3}$ Department of Chemistry, UBC, 2036 Main Mall, Vancouver, British Columbia, Canada V6T 1Z1 \\ ${ }^{4}$ Materialen Fisika Saila, Kimika Fakultatea, UPV/EHU, 1072 Posta Kutxa, 20080 Donostia, Spain
}

(Received 21 July 2003; published 11 December 2003)

\begin{abstract}
Electrochemically prepared porous alumina membranes with a pore diameter of $58 \mathrm{~nm}$ have been investigated by electron energy-loss spectroscopy in a transmission electron microscope operated at $120 \mathrm{keV}$ and 200 $\mathrm{keV}$. Energy-loss spectra are recorded for electrons traveling along the pores at different impact parameters. The dominant spectral features near the pore wall are shown to originate in the excitation of surface plasmons. Additional loss features at $7 \mathrm{eV}(8 \mathrm{eV})$ for $200 \mathrm{keV}(120 \mathrm{keV})$ are observed, whose origin is associated to Cherenkov radiation modified by the sample nanostructure. Maxwell equations are solved for different geometrical models of the target, providing a good qualitative description of the experimental spectra. Quantitative agreement is obtained by considering multiple plasmon excitation, suggesting a promising tool to investigate pore surfaces.
\end{abstract}

DOI: 10.1103/PhysRevB.68.245407

PACS number(s): 79.20.Uv, 41.60.Bq, 73.20.Mf

\section{INTRODUCTION}

Electron energy-loss spectroscopy (EELS) and energyfiltered transmission microscopy are now established as important tools for high-resolution chemical and physical characterization of new materials. ${ }^{1-3}$ The chemical information is provided by energy losses involving core electrons, in the range from 50 to $1000 \mathrm{eV}$. The low-loss spectrum below 50 $\mathrm{eV}$ is dominated by bulk and surface plasmons involving valence electrons. The typical electron beam energy used for analysis $(200 \mathrm{keV})$ corresponds to relativistic electron velocities $(v / c \approx 0.7)$, leading to retardation effects in both the low-loss and core-loss features. These effects have long been known for electrons passing directly through specimens, ${ }^{4}$ but recent theoretical work ${ }^{5}$ has shown that EELS with fast electrons traveling through vacuum near dielectric materials also exhibits relevant effects such as surface-plasmon dispersion and Cherenkov radiation. The necessity of a relativistic formulation to account for aloof EELS experiments has been claimed many times in the literature. ${ }^{6,7}$

Cherenkov radiation with frequency $\omega$ is produced when a charged particle moves through a medium at a velocity greater than the speed of light in that medium, but it can be produced even for electrons in vacuum traveling near a material, provided the Cherenkov condition is satisfied in the medium. ${ }^{8,9}$ This effect can be important up to electronsurface separations of the order of the range of the evanescent field of the electron in vacuum for the corresponding frequency component $\omega$, which is approximately given by $v / \omega$. The radiative nature of the Cherenkov effect introduces a high sensitivity to the target geometry up to much larger distances. ${ }^{10}$

Radiative losses have been observed in dielectrics and semiconductors ${ }^{11,12}$ when the electron beam passes through the material, and the optical emissions that arise due to these effects have been recently measured. ${ }^{13}$ It is only recently that the aloof Cherenkov effect has been considered, in a report on the loss spectra of alumina nanoparticles. ${ }^{14}$ The authors report features in the EEL spectrum below $10 \mathrm{eV}$, where the nonretarded theory predicts no loss. Porous silicon also shows unusual spectral features at large beam-surface separation that may be due to the Cherenkov effect. ${ }^{15}$ It is clear that nanostructured materials can exhibit interesting features in their loss spectrum.

The material under consideration in the present work (i.e., porous alumina, see Figs. 1 and 2) has many applications in material science ${ }^{16-20}$ and thin cross sections of this material have been investigated by EELS in the core-loss regime. ${ }^{21}$ We now report the low-loss spectra for electron beams traveling parallel to the pores of a porous alumina membrane, and show that they exhibit a very pronounced loss peak due to Cherenkov radiation. We follow an analytical approach to yield simple expressions for the energy-loss probability of electrons moving in nanoporous media within the framework of dielectric theory. Numerical calculations are also performed for a more detailed description of the pores, considering first- and second-neighbor pores of a central one. In this case we use the boundary element method (BEM). ${ }^{22}$ Gaussian atomic units (i.e., $\hbar=e^{2}=m=1$ ) are used throughout the paper unless otherwise specified.

\section{SAMPLE FABRICATION AND EELS MEASUREMENTS}

Porous alumina thin films were fabricated by anodizing $1.0-\mu \mathrm{m}$-thick Al films deposited onto $n$-Si(111) wafers by $e$-beam evaporation. The anodization was carried out in 0.3 $\mathrm{M}$ oxalic acid at $20^{\circ} \mathrm{C}$ with an applied potential of $40.0 \mathrm{~V}$, using a glass cell with an O-ring seal to the substrate. The resulting porous alumina film (1.4 $\mu \mathrm{m}$ thick) was detached from the $\mathrm{Si}$ wafer but still attached to the surrounding $\mathrm{Al}$ film. A 40-min etching step with $5 \mathrm{wt} \%$ phosphoric acid was used to remove the barrier layer at the bottom of the pores, and increase the pore diameter. The film was rinsed with distilled water and ethanol, and then dried with a heat gun. Small pieces were cut free with a diamond scribe and at- 


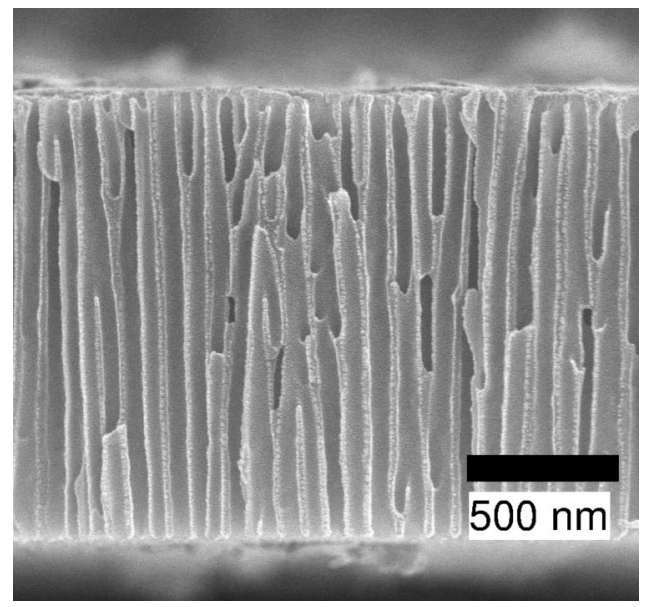

FIG. 1. SEM image showing the porous alumina film used for this study in cross section.

tached to a Cu TEM grid with a small amount of epoxy glue.

The membranes obtained in this manner showed a substantial amount of disorder in the packing and size of the pores (Fig. 1); isolated straight pores were used for analysis. Membranes with a higher degree of ordering ${ }^{23,24}$ can be obtained using a two-step anodization procedure but were not produced for this study.

Electron energy-loss spectroscopy was carried out on a Tecnai F20 TEM with a Schottky field-emission electron gun, operated at 117 and $197 \mathrm{keV}$ primary beam energies. Nevertheless, throughout the paper we will use the nominal values of $120 \mathrm{keV}$ and $200 \mathrm{keV}$, respectively. The films were imaged in scanning-tunneling electron microscopy (STEM) mode using a high-angle annular dark field detector, and loss spectra were acquired at fixed intervals across the diameter of a pore. A post-column Gatan imaging filter with a 1024channel charge-coupled device detector was used to collect the loss spectra. The detector dispersion was set to $0.05 \mathrm{eV} /$ channel, giving a $50-\mathrm{eV}$ collection range. The full width at half maximum (FWHM) of the primary beam energy distri-

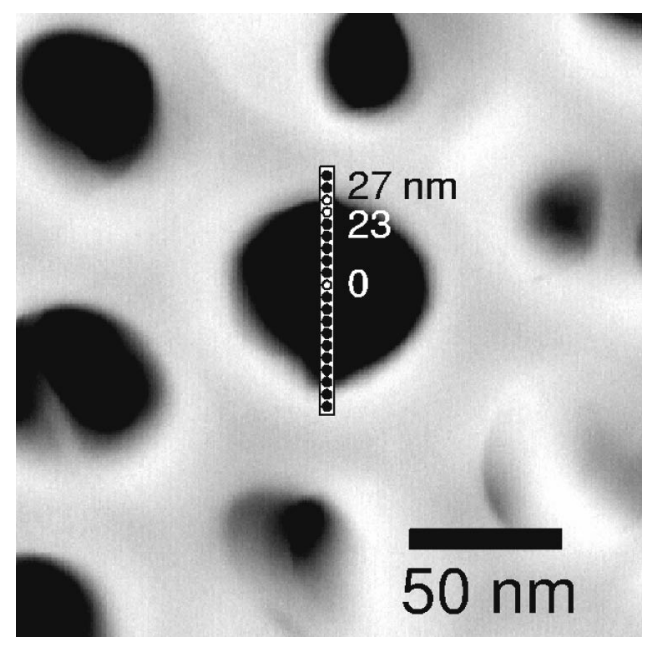

FIG. 2. Dark-field STEM image showing the porous alumina film and the locations at which loss spectra were recorded. The impact parameter $s$ of representative spectra is indicated.

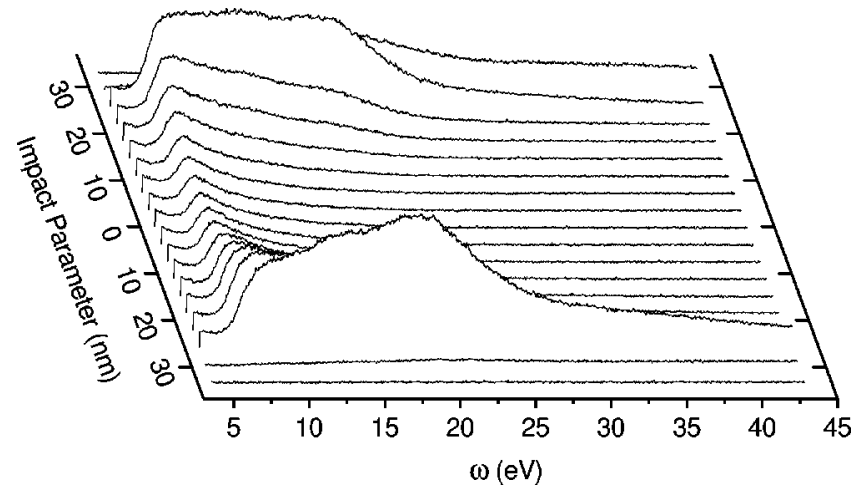

FIG. 3. Electron energy-loss spectra acquired with a 200-keV electron beam at the positions indicated in Fig. 2. The loss spectra are truncated at $4 \mathrm{eV}$ to remove the zero-loss peak.

bution was $0.85 \mathrm{eV}$ at $200 \mathrm{keV}$ and $1.0 \mathrm{eV}$ at $120 \mathrm{keV}$. The spectrometer collection angle was varied between $0.34 \mathrm{mrad}$ and $6.0 \mathrm{mrad}$ at $200 \mathrm{keV}$ and fixed at $1.5 \mathrm{mrad}$ at $120 \mathrm{keV}$. The probe size was not determined directly but estimated to be less than $1 \mathrm{~nm}$.

The spatial distribution of the loss modes was determined by acquiring loss spectra at fixed intervals across the diameter of a pore (radius $\approx 29 \mathrm{~nm}$ ) in the film. Figure 2 shows the STEM image of the membrane, along with the location of the recorded spectra. The spatial distribution of the loss spectra at $200 \mathrm{keV}$ is shown in Fig. 3. Due to the thickness of the membrane, no signal is observed outside of the pores. Near the wall, the signal is dominated by the alumina surface-plasmon contribution around $20 \mathrm{eV} .^{5}$ The shoulder above this energy can arise from bulk-plasmon excitation at $23 \mathrm{eV}$ originated by some roughness of the pores as well as from multiple plasmon losses. At larger distances from the surface, the loss spectrum is almost flat between 7 and $20 \mathrm{eV}$. Near the center of the pore, the surface plasmon loss peaks fade out and the main feature is a sharp and asymmetric peak around $7 \mathrm{eV}$. Representative spectra for these three regions are shown in Fig. 4. At a primary beam energy of $120 \mathrm{keV}$,

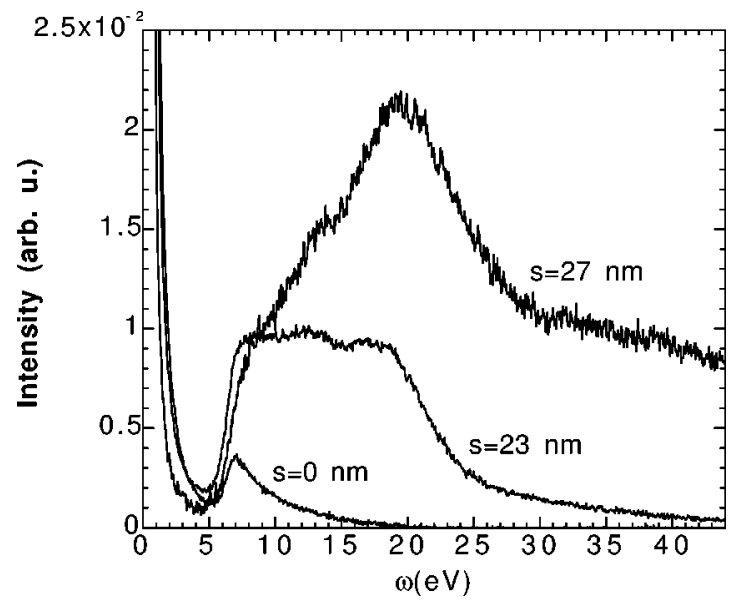

FIG. 4. Representative loss spectra for a pore in porous alumina at three different impact parameters: axial $(s=0 \mathrm{~nm})$, intermediate $(s=23 \mathrm{~nm})$, and near wall $(s=27 \mathrm{~nm})$ obtained with a $200-\mathrm{keV}$ electron beam. 


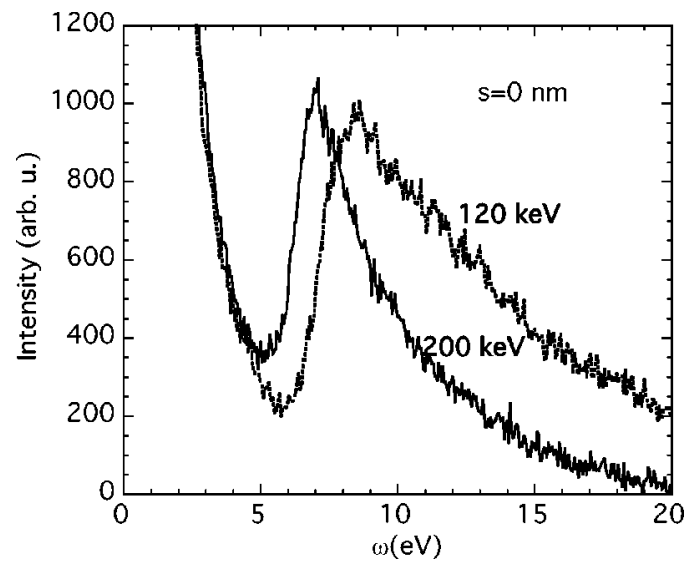

FIG. 5. Comparison of experimental loss spectra at axial trajectories $(s=0 \mathrm{~nm})$ for two different primary beam energies (solid line is for $200 \mathrm{keV}$ and dashed line for $120 \mathrm{keV}$ ).

the loss feature at the center of the pore is shifted higher by $1.5 \mathrm{eV}$ (Fig. 5). The loss feature at large distances from the surface is significant because it arises in the alumina gap, where the dielectric function $\epsilon(\omega)$ is real (see Fig. 6). The fact that the real part of $\epsilon(\omega)$ is quite large in that region suggests that Cherenkov radiation is responsible for this loss feature. Such an unusually large beam-surface interaction has also been noted for porous silicon. ${ }^{15}$

Spectra acquired with different collection angles showed little difference in the distribution of losses, in agreement with the low scattering angles for the Cherenkov effect and surface plasmons. ${ }^{3}$

Additional constraints to the interpretation of the energyloss spectra arise from the large thickness of the sample, so that for probe trajectories close to the wall, the momentum transfer becomes large enough and as a result, part of the scattered electrons could emerge out of the collection angle. In fact, the thickness of the sample restricts the actual collection angle of the experimental setup, working as an effective aperture of about $20 \mathrm{mrad}$ for axial trajectories. On the

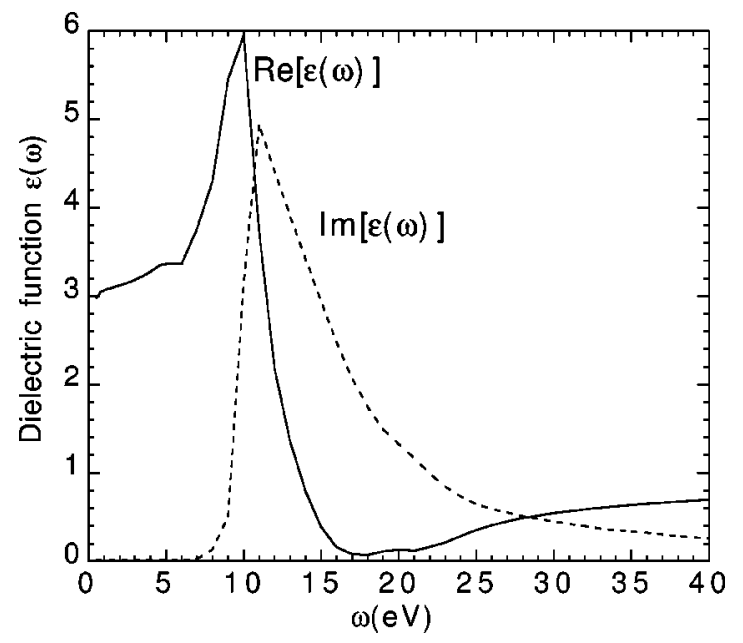

FIG. 6. Real (continuous line) and imaginary part (dashed line) of the dielectric function of alumina, as obtained from optical data (Ref. 25). other hand, the effects derived from the divergence of the beam are likely to become relevant for trajectories near the interface, where some of the electrons can get into the alumina. All these effects are important for aloof trajectories, but it is difficult to estimate them quantitatively. Anyway, the overall consequence is a reduction of the number of collected electrons, with a more pronounced reduction when the probe travels near the pore surface. Notice that this reduction is not a kinematical constraint, but rather due to the geometry of the system formed by the probe and the sample.

\section{RETARDED ENERGY-LOSS PROBABILITY FOR A SWIFT ELECTRON MOVING IN A CYLINDRICAL NANOPORE IN A POROUS MEDIUM}

We derive first a simple analytical expression for the energy loss of an electron moving along an infinitely long cylindrical shell. In fact, the magnitude we calculate is the energy-loss per unit length, but for conciseness we will use the term energy-loss probability. The reliability of this approach will be discussed later. The expression for a single hole $^{26,27}$ in an infinite medium comes as a particular case and will be considered later. As is discussed in the following section, the main advantage of the shell model is that it takes into account the Cherenkov radiation reflection in the wall of the pores through the outer cylindrical surface. Furthermore, as the total internal condition is satisfied, the radiation is confined in the cylindrical shell, as shown in Fig. 7(b). In the single-hole model, on the other hand, the radiation propagates outwards with no limitation. The actual experimental geometry, which will be considered in the following section with the BEM method, is somewhere between these cases. We consider one electron moving parallel to the axis $(z$ direction) of a hole in porous alumina, modeled as an infinitely long cylindrical shell of alumina (radii $a$ and $b$, with $a<b$ ) surrounded by a third medium (see Fig. 7). The three different media are labeled 1 (core medium), 2 (intermediate medium), and 3 (outer medium). The distance from the electron trajectory to the cylinder axis, impact parameter, is denoted by $s$. The current and charge densities associated to the swift electron are

$$
\vec{j}(\vec{r}, t)=\rho(\vec{r}, t) \vec{v}
$$

and

$$
\rho(\vec{r}, t)=-\frac{1}{r} \delta(r-s) \delta(z-v t),
$$

expressed in cylindrical coordinates $(r, \phi, z)$. We have to solve Maxwell equations for the electromagnetic field $(\vec{E}, \vec{H})$, subject to the customary boundary conditions at both cylindrical interfaces. In the region of the external electron, these fields can be separated into two contributions, $\vec{E}=\vec{E}^{H}$ $+\vec{E}^{D}$ and $\vec{H}=\vec{H}^{H}+\vec{H}^{D}$, where the label $H$ refers to the solution of the homogeneous wave equation and label $D$ indicates the particular direct solution of the wave equation with the electron source. The resulting equations (a set of eight independent equations with eight coefficients) are solved in 
a)

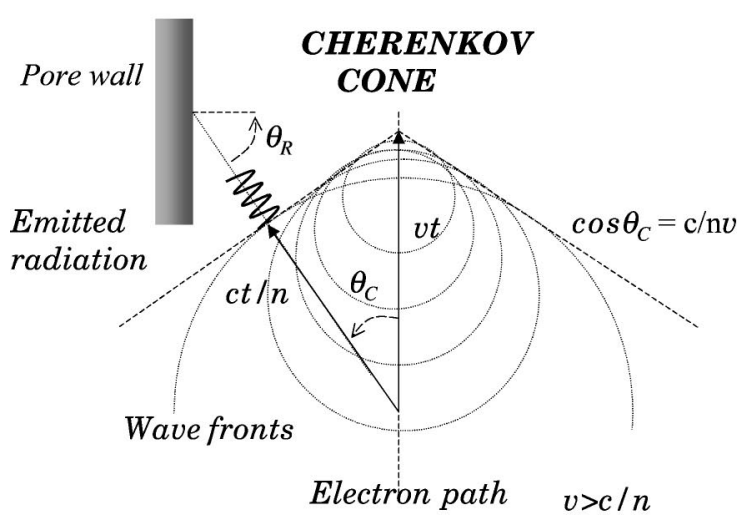

b)

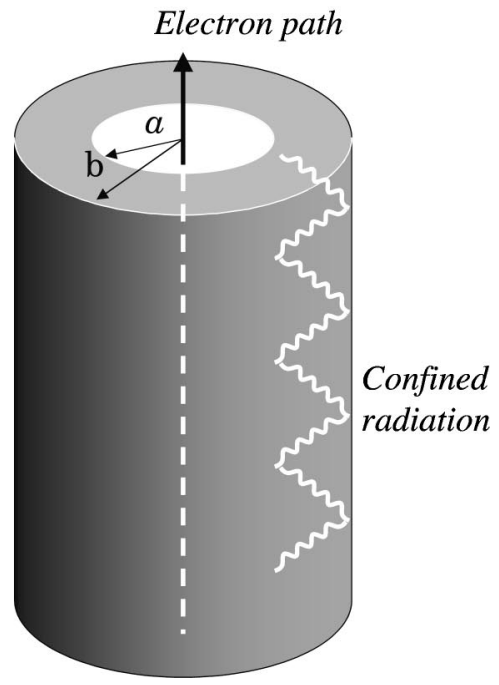

FIG. 7. (a) Cherenkov radiation emission cone due to a swift electron (velocity $v$ ) moving in an infinite medium of refractive in$\operatorname{dex} n=\sqrt{\epsilon(\omega)}$. (b) Cherenkov radiation due to the electron moving along the axis suffers internal reflection in the cylindrical shell (radii $a<b$ ) of a material when the Cherenkov condition is satisfied in that medium.
Appendix A. This permits calculation of the energy-loss probability, which is obtained from the induced force acting back on the electron along its trajectory, $\vec{r}=\vec{r}_{0}$ (i.e., the homogeneous contribution to the $z$ component of the electric field), and after solving we find

$$
F_{z}^{i n d}=-E_{z}^{i n d}\left(\vec{r}=\overrightarrow{r_{0}}\right)=\int_{0}^{+\infty} d \omega \omega \frac{d P(\omega)}{d z} .
$$

For the shell, we concentrate on the axial trajectory $s=0$ for which this expression becomes very simple, owing to the symmetry of the problem. Moreover, Cherenkov effects show up more clearly for trajectories as far as possible from the pore walls, where surface modes are not easily excited. The energy-loss probability per unit length is then

$$
\frac{d P(\omega)}{d z}=\frac{2}{\pi v^{2}} \operatorname{Im}\left\{\frac{1-\epsilon_{1}(\omega) \beta^{2}}{\epsilon_{1}(\omega)} F(\omega)\right\},
$$

where

$$
F(\omega)=\frac{F_{12}(\omega)+F_{23}(\omega) G_{12}(\omega)}{1+F_{23}(\omega) H_{12}(\omega)},
$$

and

$$
F_{12}=\frac{\epsilon_{2} \nu_{1} K_{0}\left(\nu_{1} a\right) K_{0}^{\prime}\left(\nu_{2} a\right)-\epsilon_{1} \nu_{2} K_{0}^{\prime}\left(\nu_{1} a\right) K_{0}\left(\nu_{2} a\right)}{\epsilon_{2} \nu_{1} I_{0}\left(\nu_{1} a\right) K_{0}^{\prime}\left(\nu_{2} a\right)-\epsilon_{1} \nu_{2} I_{0}^{\prime}\left(\nu_{1} a\right) K_{0}^{\prime}\left(\nu_{2} a\right)},
$$

$$
\begin{gathered}
F_{23}=\frac{\epsilon_{3} \nu_{2} K_{0}\left(\nu_{2} b\right) K_{0}^{\prime}\left(\nu_{3} b\right)-\epsilon_{2} \nu_{3} K_{0}^{\prime}\left(\nu_{2} b\right) K_{0}\left(\nu_{3} b\right)}{\epsilon_{3} \nu_{2} I_{0}\left(\nu_{2} b\right) K_{0}^{\prime}\left(\nu_{3} b\right)-\epsilon_{2} \nu_{3} I_{0}^{\prime}\left(\nu_{2} b\right) K_{0}\left(\nu_{3} b\right)} \\
G_{12}=\frac{\epsilon_{1} \nu_{2} I_{0}\left(\nu_{2} a\right) K_{0}^{\prime}\left(\nu_{1} a\right)-\epsilon_{2} \nu_{1} I_{0}^{\prime}\left(\nu_{2} a\right) K_{0}\left(\nu_{1} a\right)}{\epsilon_{2} \nu_{1} I_{0}\left(\nu_{1} a\right) K_{0}^{\prime}\left(\nu_{2} a\right)-\epsilon_{1} \nu_{2} I_{0}^{\prime}\left(\nu_{1} a\right) K_{0}^{\prime}\left(\nu_{2} a\right)} \\
H_{12}=\frac{\epsilon_{1} \nu_{2} I_{0}\left(\nu_{2} a\right) I_{0}^{\prime}\left(\nu_{1} a\right)-\epsilon_{2} \nu_{1} I_{0}^{\prime}\left(\nu_{2} a\right) I_{0}\left(\nu_{1} a\right)}{\epsilon_{2} \nu_{1} I_{0}\left(\nu_{1} a\right) K_{0}^{\prime}\left(\nu_{2} a\right)-\epsilon_{1} \nu_{2} I_{0}^{\prime}\left(\nu_{1} a\right) K_{0}^{\prime}\left(\nu_{2} a\right)}
\end{gathered}
$$

Here, $I_{0}$ and $K_{0}$ are the modified Bessel functions of zero order and $I_{0}^{\prime}$ and $K_{0}^{\prime}$ stand for their derivatives with respect to the argument. The functions that multiply the radii in the arguments are $\nu_{i}=(\omega / v) \sqrt{1-\epsilon_{i}(\omega) \beta^{2}}$, where the index $i$ $=1,2,3$ refers to the different cylindrical regions, and $\beta$ $=v / c$. Note that if the limit $\nu_{1}=\nu_{2}$ is taken then $F_{12}=0$, $G_{12}=1, H_{12}=0$, and $F(\omega)=F_{23}$. In this way, we recover the expression corresponding to a cylindrical hole of dielectric constant $\epsilon_{1}$ in an infinite medium of dielectric constant $\epsilon_{3}$, which has been already given in the past. ${ }^{27}$ In the same way, by taking $\nu_{2}=\nu_{3}$ we get $F(\omega)=F_{12}$, the expression for a cylindrical hole of dielectric constant $\epsilon_{1}$ in an infinite medium of dielectric constant $\epsilon_{2}$. The nonretarded expression for the cylindrical shell is recovered in the limit $\beta \rightarrow 0$ from Eq. (4). The retarded energy-loss probability for an electron moving in a cylindrical hole parallel to its axis was obtained $^{27}$ previously for any impact parameter $s$. It is given by expression (4) with

$$
F(\omega)=\sum_{m=0}^{\infty}\left(2-\delta_{m 0}\right) I_{m}^{2}\left(\nu_{1} s\right) \frac{a \epsilon_{2} \nu_{1} K_{m}\left(\nu_{1} a\right) K_{m}^{\prime}\left(\nu_{2} a\right)-\epsilon_{1} \nu_{2} K_{m}^{\prime}\left(\nu_{1} a\right) K_{m}\left(\nu_{2} a\right)-\eta_{m} I_{m}\left(\nu_{1} a\right) K_{m}\left(\nu_{1} a\right) K_{m}^{2}\left(\nu_{2} a\right)}{a \epsilon_{2} \nu_{1} I_{m}\left(\nu_{1} a\right) K_{m}^{\prime}\left(\nu_{2} a\right)-\epsilon_{1} \nu_{2} I_{m}^{\prime}\left(\nu_{1} a\right) K_{m}\left(\nu_{2} a\right)-\eta_{m} I_{m}^{2}\left(\nu_{1} a\right) K_{m}^{2}\left(\nu_{2} a\right)}
$$


and

$$
\eta_{m}=\frac{m^{2} \beta^{2}(\omega / v)^{4}\left(\epsilon_{1}-\epsilon_{2}\right)^{2}}{\nu_{1}^{2} \nu_{2}^{2}\left[\nu_{1} I_{m}\left(\nu_{1} a\right) K_{m}^{\prime}\left(\nu_{2} a\right)\right]-\nu_{2} I_{m}^{\prime}\left(\nu_{1} a\right) K_{m}\left(\nu_{2} a\right)} .
$$

The expression for nonaxial trajectories is a sum of different $m$ terms, where the modified Bessel functions of order $m$ $\left(I_{m}, K_{m}\right)$ and their derivatives appear. The axial case $s=0$ is considerably simpler due to the vanishing of terms $m \neq 0$, because $I_{m}^{2}\left(\nu_{1} s\right)$ is zero when $s=0$ except for $m=0$, in that case $I_{0}\left(\nu_{1} 0\right)=1$. Note that in the axial case, Eq. (10) gives expression (5) with $\epsilon_{2}=\epsilon_{3}$. In general, the dielectric functions $\epsilon_{1}(\omega), \epsilon_{2}(\omega)$, and $\epsilon_{3}(\omega)$ are complex, so that the Bessel functions have complex-number arguments. However, in order to study the Cherenkov effect, and to understand the underlying physics, it is instructive to use a real dielectric function, in which case these arguments are either real or imaginary. The energy-loss probability then becomes a superposition of Dirac $\delta$ functions (see Appendix B):

$$
\frac{d P(\omega)}{d z}=\sum_{i} f(\omega) \delta\left(\omega-\omega_{i}\right),
$$

where the energies $\omega_{i}$ of the Cherenkov modes are given by the zeros of a real function given in Appendix B. The function $f(\omega)$ in also obtained there. Assuming that both surrounding media are perfectly reflective $\left(\epsilon_{1}=\epsilon_{2}=\infty\right)$, one can obtain an approximate expression for these modes:

$$
\omega \simeq \frac{n \pi v}{(b-a) \sqrt{\beta^{2} \epsilon_{2}-1}},
$$

with $n=1,2,3, \ldots$. Note that this expression is only valid in the alumina gap.

\section{A. Results for a single pore}

As a first approach to describe the porous membrane when the electron travels along one pore, we consider the simplest possibility, a single hole in alumina (model $A$ ). The energy-loss probability is then given by expressions (4) and (10). This was used in the literature ${ }^{27}$ to explain EELS data obtained for a hole drilled in aluminum fluoride and alumina using a STEM. ${ }^{28}$ In Fig. 8 we show the results for the energy-loss probability calculated for $200-\mathrm{keV}$ electrons in a hole of radius $a=29 \mathrm{~nm}$ in alumina. We have considered three different impact parameters, namely, $s=0,23 \mathrm{~nm}$, and $27 \mathrm{~nm}$, in order to compare with the experimental spectra, using the available optical data for the dielectric function of alumina. $^{25}$ There are some plasmon features between $10 \mathrm{eV}$ and $25 \mathrm{eV}$, to be compared with the nonretarded planar surface losses, proportional to $\operatorname{Im}\left[\left(\epsilon_{2}-1\right) /\left(\epsilon_{2}+1\right)\right]$, which in alumina presents a peak around $\omega_{s}=20 \mathrm{eV}$.

In addition to these surface-plasmon features, the three spectra present significant losses below $10 \mathrm{eV}$, which as discussed above, come from retardation effects associated with Cherenkov radiation. Moreover, the real part of the dielectric function of alumina is large enough to fulfill the Cherenkov condition $v^{2} / c^{2}>1 / \operatorname{Re}(\epsilon)$. For planar interfaces, retardation

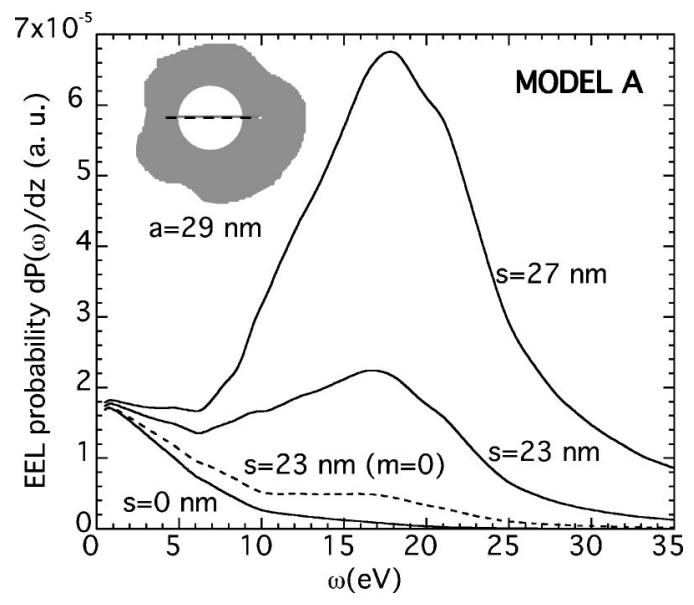

FIG. 8. Comparison of calculated energy-loss spectra for a 200$\mathrm{keV}$ electron in a single hole of radius $a=29 \mathrm{~nm}$ in alumina and three different impact parameters: $s=0$ (axial), $s=23 \mathrm{~nm}$ (intermediate), and $s=27 \mathrm{~nm}$ (close to the edge). For the intermediate impact parameter the contribution of the $m=0$ mode has been plotted with small dashes.

introduces a dispersion, which is reflected in a redshift of the surface loss peak as the impact parameter increases. ${ }^{5,7}$ In addition to this impact-parameter-dependent dispersion, the modes of a cylindrical cavity exhibit a dispersion related to the azimuthal numbers $m$. Nevertheless, for such a large pore $\left(\omega_{s} a / v \gg 1\right)$, all the nonretarded modes appear at $\omega_{s},{ }^{27}$ and therefore the dispersion shown in this case is basically a relativistic effect. The surface-plasmon losses are higher as the beam approaches the interface, as explained by the classical dielectric theory, which for a flat surface predicts that the energy-loss probability per unit length goes as $K_{0}(2 \omega b / v)$ ( $b$ is the impact parameter relative to the surface). ${ }^{29}$ In the cylindrical case, this enhancement near the interface is obtained by increasing contributions of many azimuthal $m$ modes. In Fig. 8, we have plotted the contribution of the $m=0$ mode to the loss spectrum corresponding to a small impact parameter $(s=23 \mathrm{~nm})$ relative to the pore wall, and here we see that it provides approximately $20 \%$ of the total plasmon excitation probability. The contribution of the other modes has been studied elsewhere. ${ }^{30}$

Furthermore, for the three impact parameters considered in the figure, Cherenkov losses present a much weaker dependence on the impact parameter. In fact, the three spectra reach the same limit as $\omega \rightarrow 0$. This value is exactly the same one corresponding to Cherenkov emission in an unbounded medium: ${ }^{31}$

$$
\frac{d P(\omega)}{d z}=\frac{1}{c^{2}}\left\{1-\frac{1}{\beta^{2} \operatorname{Re}(\epsilon)}\right\}
$$

which for alumina gives just $1.7 \times 10^{-5}$ a.u. The independence of the Cherenkov loss intensity on impact parameter in this region is a consequence of the large wavelength $\lambda$ of the associated radiation. That is, when $\lambda \gg a$ then the geometrical parameters $a$ and $s$ become irrelevant. This different impact-parameter dependence in both Cherenkov and plasmon regions of the spectra is clearly illustrated in Fig. 8. 
Although this simple model explains the two different mechanisms of inelastic excitations found in experiments, it fails to reproduce the peak associated to Cherenkov radiation around $7 \mathrm{eV}$. The origin of this peak seems to be related to the coupling of the central pore to the surrounding ones.

One simple way to introduce the effect of this coupling inside this simple model is to represent the dielectric response of the membrane through an effective dielectric function. We can use, for example, the well-known MaxwellGarnett formula, ${ }^{32}$ generally used to treat dilute systems of particles with dielectric function $\epsilon_{1}$ in a medium of dielectric function $\epsilon_{2}$. If $f$ is the volume fraction of component 1 (the vacuum inside cylindrical pores) inside component 2 (the alumina), the effective dielectric function is given by

$$
\epsilon_{e f f}=\epsilon_{2}+\frac{f \epsilon_{2}\left(\epsilon_{1}-\epsilon_{2}\right)}{\epsilon_{2}+(1-f)\left(\epsilon_{1}-\epsilon_{2}\right) / 2},
$$

where $\epsilon_{1}=1$ and $\epsilon_{2}$ corresponds to alumina. This formula is valid for the case of cylindrical particles, avoiding the effects of the borders. Thus its validity is limited to the situation where the radii of the holes are much smaller than the sample thickness. This model gives a loss maximum at around 9-10 $\mathrm{eV}$ when choosing for instance $f \approx 0.4$, but it is unable to fit both Cherenkov and plasmon parts of the spectra simultaneously. In fact, within this effective medium model, the membrane is considered homogeneous, and all diffraction effects characteristic of the propagation of light in inhomogeneous media are neglected.

\section{B. Results for a cylindrical shell}

In order to describe the features associated with the inhomogeneity of the sample, we consider a cylindrical alumina shell of radius $b$ surrounding the central hole in which the electron moves (model $B$ ). We have considered an inner radius $a=29 \mathrm{~nm}$, which is the average radius of the hole marked in the micrograph of Fig. 1. For the outer radius three different values are considered, $b=61,94$, and $127 \mathrm{~nm}$. These radii have also approximately been chosen from the micrograph and correspond to an average minimum distance from the center of the central hole to the surface of the closest six pores, to the second closest six pores, and the mean value of these two distances.

To compare with the simple hole model studied above, we have plotted in Fig. 9 the corresponding EEL spectrum for axial trajectories with the same radius $a$ as in Fig. 8. First of all, one can see that the spectra corresponding to the shell are in good mutual agreement within the surface-plasmon region. This is because the coupling between both surfaces of the shell is governed by the range of the $\omega$ component of the Coulomb potential, which neglecting retardation, is given by $v / \omega$ (about $9 \mathrm{~nm}$ for $\omega=15 \mathrm{eV}$ ). Then, above $\omega=10 \mathrm{eV}$, this coupling is negligible in the actual problem, as well as in the current model for the radii under consideration. The differences with respect to the single-pore case show up dramatically in the Cherenkov emission region. There, the spectra consist of a set of very sharp peaks, and therefore, no radiation except for the discrete frequencies of Eq. (13). The width of the loss peaks below $7 \mathrm{eV}$ are a consequence of the

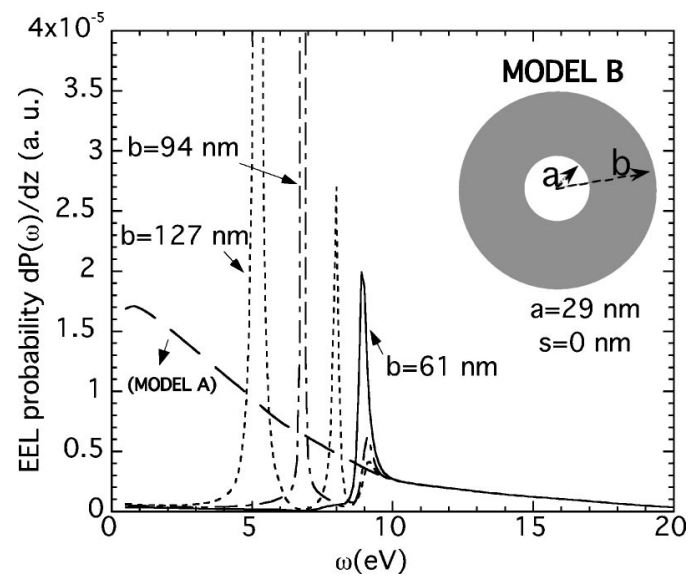

FIG. 9. Calculated EEL spectra for 200-keV electrons traveling along the axis (impact parameter $s=0$ ) of a cylindrical shell of alumina of inner radius $a=20 \mathrm{~nm}$ and different external radii, $b$ $=61,94$, and $127 \mathrm{~nm}$. The spectrum corresponding to a single hole of radius $a=29 \mathrm{~nm}$ in alumina has been plotted for comparison.

small imaginary part (0.01) that we have added to the dielectric function in order to facilitate the visualization of these peaks. For the thinnest shell $(b=61 \mathrm{~nm})$, only one sharp peak appears around $9 \mathrm{eV}$, whereas for $b=94 \mathrm{~nm}$ a second peak shows up, and for the thickest shell $(b=127 \mathrm{~nm})$ there are three peaks. Thus, we see that more peaks emerge as the thickness increases, so that the lower energy peak is the most intense. The intermediate thickness is the one that best reproduces the experimental peak, since the position of the lowest energy loss peak is around $7 \mathrm{eV}$, similar to the measured one (Fig. 4). These features of the EEL spectra are a consequence of the reflection of the radiation on the surfaces of the shell, which behaves like a waveguide that confines the radiation.

In fact, the condition for total internal reflection [i.e., $\sqrt{\epsilon_{2}} \sin \left(\theta_{R}\right) \geqslant 1$, where the incidence angle $\theta_{R}$ for this geometry is just the complementary angle of the Cherenkov radiation angle, $\theta_{R}=\pi / 2-\theta_{C}$; see Fig. 7] is automatically fulfilled for Cherenkov radiation. The confinement of Cherenkov radiation by total reflection in dielectric cylinders is well known. ${ }^{33}$ In the cylindrical shell geometry, the reflection on the surfaces of the shell leads to quantization of the component of the radiation momentum normal to the velocity $v$. This effect is shown in Fig. 10, where we study the dependence of the loss peak energy $\omega$ as a function of shell thickness $(b-a)$. To illustrate the simple geometrical rule of this quantization we plot the inverse length $\pi /(b-a)$ versus the normal component of the momentum $q_{\perp}=\omega\left(\beta^{2} \epsilon_{2}\right.$ $-1)^{1 / 2} / v$. Here we see that the perpendicular momentum of the allowed radiation satisfies $q_{\perp}(\omega)=m \pi /(b-a)$, where $m=1,2,3, \ldots$ is an integer. Then, the loss peaks appear at the energies given by Eq. (13); that is an approximate dispersion relation for the radiative modes with shell thickness. In Fig. 11, we study the weight of these radiative modes in the EELS spectra corresponding to an alumina shell. With a constant internal radius $a=29 \mathrm{~nm}$, the effect of varying the outer radius $b$ from $2 a$ to $180 a$ is shown for losses over $0-10 \mathrm{eV}$. As the outer radius $b$ increases, more Cherenkov modes show up in the spectrum, as predicted by Eq. (13), so 


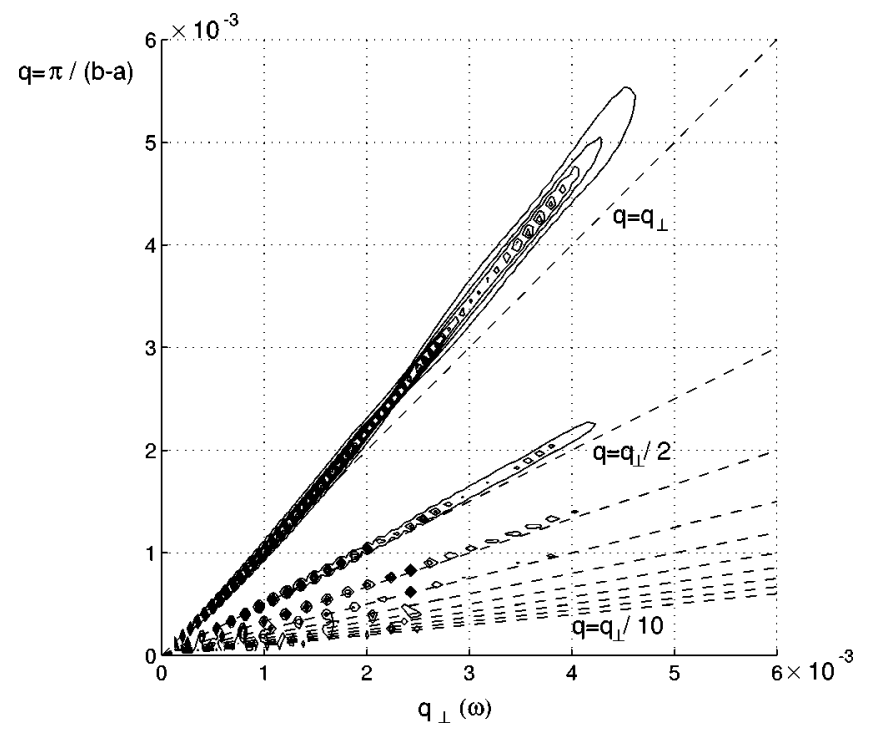

FIG. 10. Contour plots of the spectra shown in Fig. 11, but in terms of momentum; perpendicular momentum $q_{\perp}=\omega\left(\beta^{2} \epsilon_{2}\right.$ $-1)^{1 / 2} / v$ versus momentum $q=\pi /(b-a)$. Lines $q_{\perp}$ $=q, 2 q, 3 q, \ldots$ have been plotted as well. Atomic units are used for both.

that the limit of a single pore (Fig. 13), where the spectrum is continuous, is recovered as the separation between modes, $\pi v /(b-a) /\left(\beta^{2} \epsilon_{2}-1\right)^{1 / 2}$, goes to zero. Nevertheless, Fig. 11 shows that in this region, the spectrum is very sensitive to the width of the shell: the discrete nature of the spectrum is clear even for an outer radius of about $5 \mu \mathrm{m}$ (the largest value in this figure). This is a consequence of the propagating nature of Cherenkov radiation, which makes it sensitive to the structure of the medium far away from the probe.

As we have seen, the emission of Cherenkov radiation is a relativistic effect and depends on the energy of the incident beam. In Fig. 12, we compare the EELS spectra for two different values of incident electron energy (considered in Fig. 5), corresponding to $\beta=0.7$ and $\beta=0.6$. For larger $\beta$, the peaks increase in intensity and move towards lower energies. This behavior is also observed in the experiment,

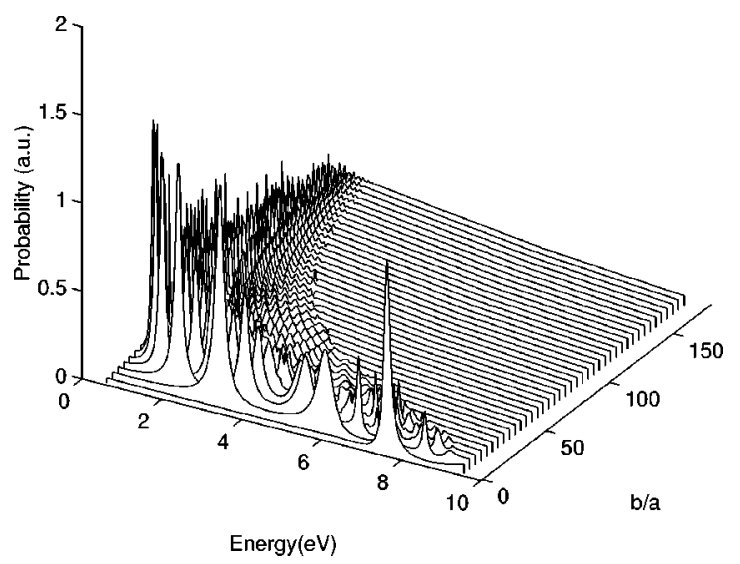

FIG. 11. Calculated EEL spectra for an axial 200-keV electron in a shell of alumina, with inner radius $a=29 \mathrm{~nm}$ as a function of the outer relative radius $b / a$.

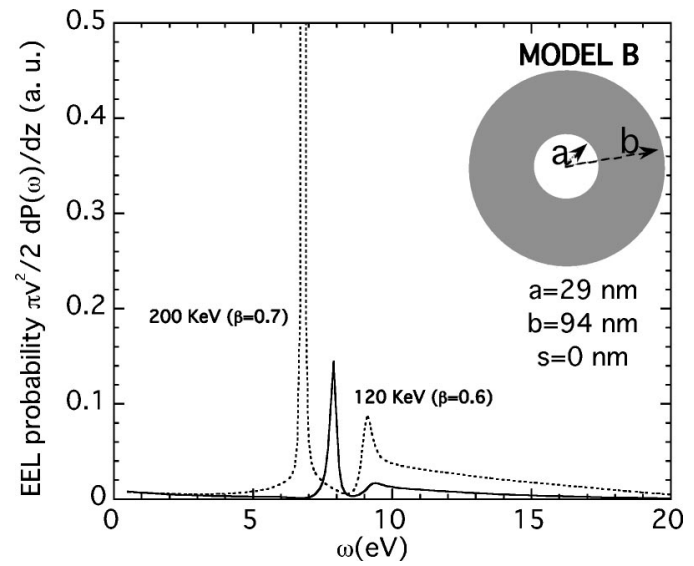

FIG. 12. Calculated EEL spectra for an electron traveling along the axis (impact parameter $s=0$ ) of a cylindrical shell of alumina of inner radius $a=29 \mathrm{~nm}$ and external radius $b=94 \mathrm{~nm}$. Two different beam energies have been considered corresponding to $\beta=0.6$ and $\beta=0.7$.

where there is not enough resolution to distinguish the two peaks, so that a single peak with a wide tail towards higher energy $\omega$ and a sharp decay in the lower energy region is observed. This result supports the Cherenkov mechanism as the origin of the observed 7-eV peak.

A better fit to experiment is obtained within this simple shell model when an effective medium is considered around the shell. That medium is represented by the effective dielectric function $\epsilon_{\mathrm{eff}}$ corresponding to alumina with cylindrical holes, obtained from Eq. (15). In this way, we are able to reproduce the position of the experimental 7-eV peak (Fig. 4) with great accuracy. In Fig. 13, we consider a shell of radii $a=29 \mathrm{~nm}$ and $b=61 \mathrm{~nm}$ for an axial electron trajectory. For the medium surrounding the alumina shell we have consid-

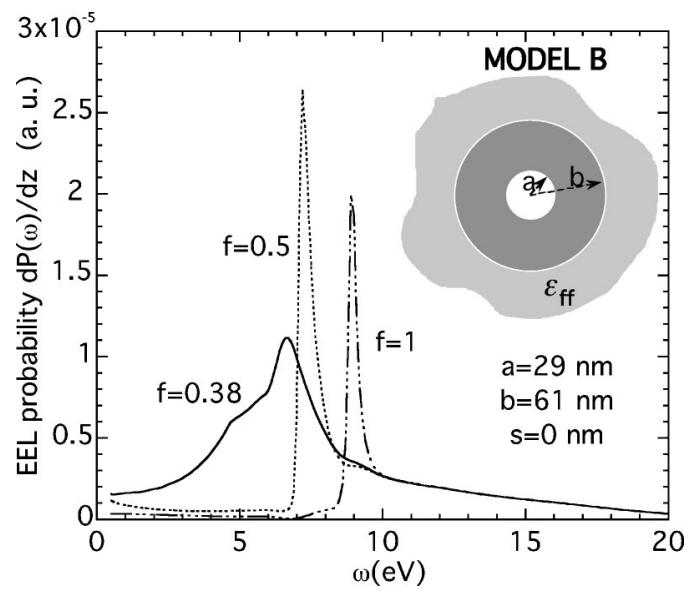

FIG. 13. Calculated EEL spectra for 200-keV electrons traveling along the axis (impact parameter $s=0$ ) of a cylindrical shell of alumina of inner radius $a=29 \mathrm{~nm}$ and outer radius $b=61 \mathrm{~nm}$, surrounded by an effective medium of alumina with cylindrical pores (see expression in the text). Three different filling fractions have been considered $(f=0.38,0.5$, and 1$)$. The last one corresponds to the limit when the effective medium is alumina, as considered in Fig. 9. 
ered an effective porous medium of different filling fractions of vacuum, $f=0.38,0.5$, and 1 , respectively. The case $f$ $=1$ corresponds to vacuum and has been included in the plot for reference. The filling fraction $f=0.38$ corresponds exactly to the pore radius and separation considered. A fraction around $f=0.4$ provides a good fit to experiment that permits reproducing the peak at $7 \mathrm{eV}$.

In conclusion, despite its simplicity, the shell model presented in this section captures the main physics that underlies the experiment: when the electron moves along a hole, Cherenkov radiation is emitted outwards and confined in the medium due to total internal reflection in the cylindrical walls. The position of the peaks in the EELS spectrum then obeys a quantization rule that involves the thickness of the cylindrical shell.

In the actual sample, the walls of the neighboring pores act as radiation reflectors that are represented by the outer cylindrical wall of the shell model. However, the radiation is not completely confined, but propagates throughout the porous medium that extends to very large distances. In the following section we consider a more realistic approach to describe the pore structure of the sample.

\section{LOSS PROBABILITY IN A PORE SURROUNDED BY HEXAGONAL PORE DISTRIBUTIONS (MODEL $C$ )}

A more realistic description of the sample is one in which the main pore (through which the electron travels) is surrounded by other pores disposed in an hexagonal fashion (model $C$ ). We consider here first- and second-neighboring pores. This problem is too complex to be solved analytically, so we have relied on numerical simulation of the Maxwell equations to solve it. In particular, the BEM has been used, ${ }^{22}$ which permits solving the electromagnetic problem in the presence of arbitrarily shaped interfaces by employing interface charge and current distributions that are solved selfconsistently for a given external field by applying the customary boundary conditions for the fields. One finds a linear set of integral equations, with the interface currents and charges as the unknowns, which are discretized by considering a set of representative points on the interfaces. More details about this method and its application to EELS can be found elsewhere. ${ }^{22}$ In the present case where the geometry is invariant for translation along the pores, the surface integrals become one-dimensional and the entire problem involves the inversion of a reduced set of $N \times N$ matrices, where $N$ is the number of discretization points. Typically, 50 points per cylinder are enough to reach convergence.

Making use of this formalism we have computed the energy-loss per unit length for two different geometries, as sketched in Fig. 14: (i) a hole surrounded by six holes forming an hexagonal distribution and (ii) a hole surrounded by 12 holes arranged in a triangular lattice. We have also considered higher-order neighbor pores, forming the crystalline structure, but the results did not introduce significant differences with respect to the ones presented here. So, we have concluded that the main physics of the problem can still be described by means of these simple models. The electronbeam energy is $200 \mathrm{keV}$, the hole radii are $a=29 \mathrm{~nm}$, and
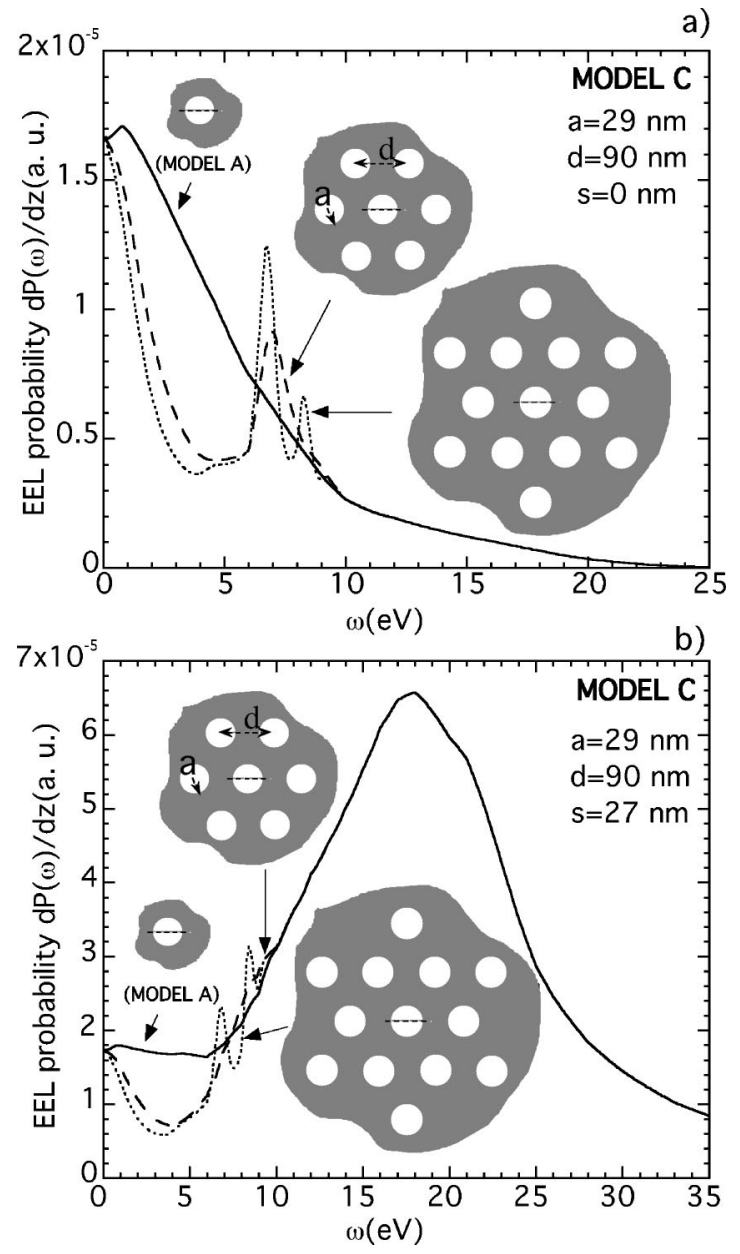

FIG. 14. Calculated EEL spectra using the BE method for a 200-keV electron in a hole of radius $a=29 \mathrm{~nm}$ in alumina (continuous line), in the same hole but surrounded by six similar holes, separated a distance $d=90 \mathrm{~nm}$ and disposed in a hexagonal way (long dashes) and in the same hole but this time considering also the second neighbors of the hexagonal lattice, at a distance $\sqrt{3} d$ from the central hole (short dashes). The impact parameters are axial ( $s$ $=0)$ in (a) and close to the wall $(s=27 \mathrm{~nm})$, in (b).

the distance between cylinder centers is $d=90 \mathrm{~nm}$. We have considered impact parameters $s=0$ (axial) and $s=27 \mathrm{~nm}$. As a reference, we have added the corresponding EEL probability for the isolated hole, the same plot as in Fig. 8. A common feature of the three plots is that they are nearly identical in the plasmon excitation energy region above $10 \mathrm{eV}$. The reason for this has been discussed above, in Sec. III. It is in the Cherenkov emission energy range $(\omega<10 \mathrm{eV})$ where the major differences are found.

We focus our study on this low-energy region. Here the spectra exhibit some features of both simple models previously considered. For small $\omega \rightarrow 0$ they show the same asymptotic behavior as the single pore, given by Eq. (14), whereas on the other hand they show peaks around the same positions as in the shell model. These two different behaviors can be understood in terms of the radiation wavelength $\lambda$ relative to the pore dimensions: above $\omega=5 \mathrm{eV}, \lambda$ is smaller than the hole diameter and therefore the transmission 
through the hole is small. In this case, reflection modes are dominant. However, below this energy range, transmission becomes favored, and therefore, radiation leaking out of the pores is the main mechanism for energy-loss. This result is likely to explain the fact that reflection on the secondneighbor holes does not lead to loss peaks around $4 \mathrm{eV}$, as predicted by the shell model. The length associated to the transverse component of the momentum would be now much larger than the diameter of the pores and therefore the reflection is weak. Finally, when $\omega \rightarrow 0$ the size of the scatterers is irrelevant as compared with the wavelength and one recovers the infinite medium limit. The fact that the two sharp loss peaks appear around the same positions as the peaks predicted by the shell model supports this argument. It also explains the reduction of the loss probability in the $1+12$ pore model, in relation to the $1+6$ pore case, as an effect of the radiation transmission through the second row of neighbors.

The energy-loss probability per unit length calculated numerically for the triangular pore distribution reproduces reasonably well the main features found in experiments (Fig. 4). However, this model fails to explain the wide structure that appears well above the surface-plasmon peak, which could originate in (1) multiple plasmon excitation as well as in (2) bulk losses due to the roughness of the walls of the pore cavity.

In order to account for the finite experimental energy resolution, the calculated data have been convoluted with the actual zero-loss peak in the experimental setup. Moreover, the effect of multiple successive losses has also been included by convoluting the resulting calculated spectra over the path length $L \sim 1.4 \mu \mathrm{m}$ similar to previous ion energyloss studies. ${ }^{34}$ In this way, an energy-loss probability along the entire trajectory is obtained using as input the loss probability per unit of path length shown in Fig. 14. The result of this analysis has been compared with experimental data in Fig. 15 for two different impact parameters. The experimental data correspond to those shown in Fig. 4. The calculation is made for the $1+6$ pore system by means of the BEM (Ref. 22) for a pore radius $a=29 \mathrm{~nm}$ and a distance between the centers of the pores equal to $90 \mathrm{~nm}$. Both experimental and simulated loss spectra have been normalized to unity. For the trajectory close to the wall, the experimental spectrum extends well above $45 \mathrm{eV}$, and the former normalization is unsuitable. In this case the experimental spectrum has been normalized so that at $\omega=0 \mathrm{eV}$ it is coincident with the simulated one.

Although all the spectra are qualitatively in agreement, close to the pore wall they are remarkably similar. In the case of the spectra closest to the wall, the effect of the multiple plasmon excitation is very clear; producing a shoulder around twice the surface-plasmon energy that was not present in the calculated energy-loss probability per unit length [Fig. 15(b)]. No relevant contribution from bulk losses is needed to explain the experimental spectra, a fact that indicates that the electron does not penetrate into the alumina and therefore that the wall of the investigated pore is quite smooth. A small difference in the energy of the observed surface peak with respect to the ideal planar surface
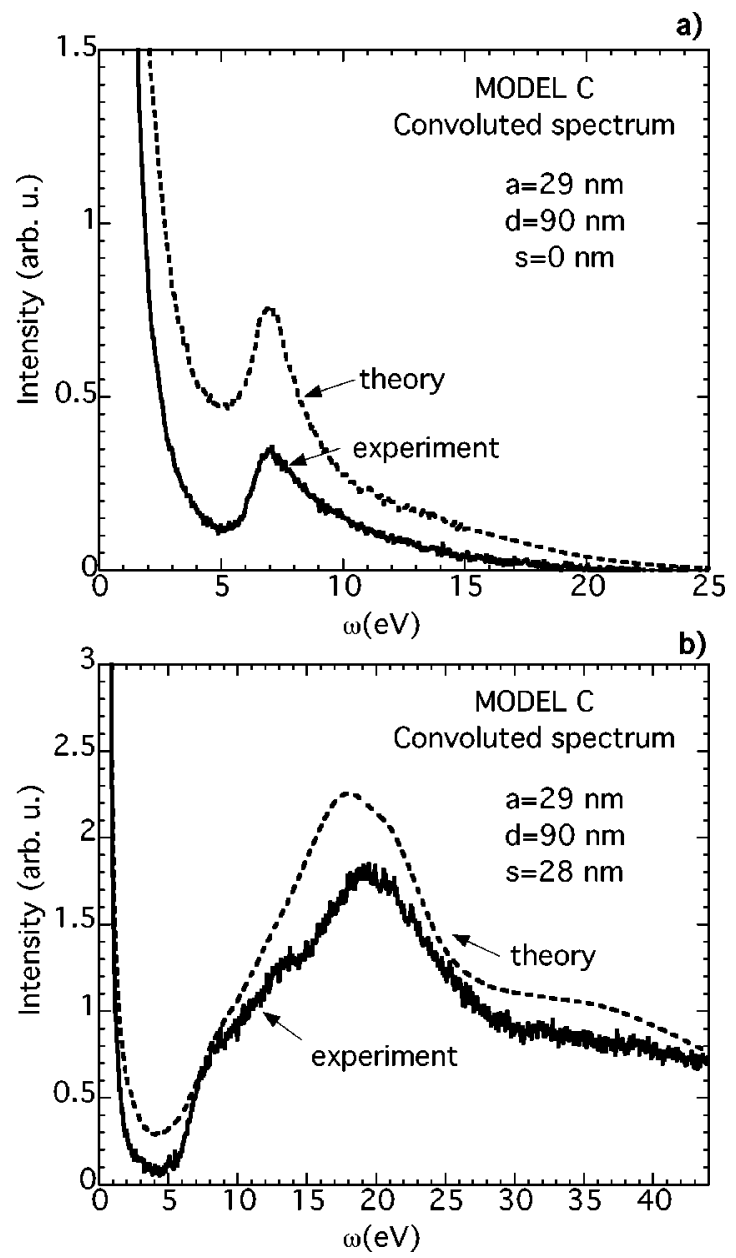

FIG. 15. Normalized energy-loss probability compared to the experimental spectra. The theoretical spectra have been obtained for the $1+6$ pore geometry $(d=90 \mathrm{~nm}, a=29 \mathrm{~nm})$ and impact parameters $s=0 \mathrm{~nm}$ (a) and $s=28 \mathrm{~nm}$ (b). Then, they have been convoluted through the pore length $L=1.4 \mu \mathrm{m}$ and normalized such that the area under each curve is the same and the heights of the zeroloss peaks are coincident. The theory has been convoluted with a measured 0.86-eV FWHM elastic peak.

can be due to the sensitivity of the alumina dielectric function to the crystalline structure, ${ }^{35}$ the defects of the sample, the curvature of the central pore, and to a minor extent, the interaction between neighboring pores. In all cases, the simulated spectra overestimate the losses below $5 \mathrm{eV}$. One possible origin of this discrepancy could be the finite length of the cavity, which does not allow Cherenkov radiation to propagate out of the sample. Above $5 \mathrm{eV}$, the existence of a small imaginary part in the dielectric function would provide a damping mechanism due to absorption of Cherenkov radiation, while below $5 \mathrm{eV}$, the imaginary part of the dielectric function is negligible and therefore the radiation is inhibited. Furthermore at $7 \mathrm{eV}$, the ratio of the thickness to the wavelength is $L / \lambda=8$, but some contribution of finite-thickness effects could still be expected at smaller energies (i.e., larger wavelengths). This fact questions the reliability of the very concept of energy loss per unit length used in our theoretical model. For surface-plasmon excitations it is well known ${ }^{36}$ that the effect of the target borders is ruled by the adiabatic 
length $v / \omega(20 \mathrm{~nm}$ for $7 \mathrm{eV})$, and therefore this effect is negligible. Nevertheless, in the case of Cherenkov radiation the nonevanescent nature of the field makes it sensitive to the size of the targets at larger distances, and one should speak properly of transition radiation. The spectra corresponding to the axial trajectory show less quantitative agreement, in both plasmon and Cherenkov loss regions. Clearly, for this trajectory the effects of the finite thickness of the membrane should be more relevant. In fact, the study of EELS in planar interfaces shows that the effect of retardation is to increase the loss probability, and this is more relevant as the impact parameter increases. For large impact parameters, the main contribution to the spectrum arises from very small momentum transfer, so that they are likely to be very sensitive to the target shape at large distances. ${ }^{5}$ In our problem it means that we are overestimating the retardation effects, obtaining an EEL probability larger than the measured one.

\section{CONCLUDING REMARKS}

We have reported EELS analysis of nanoporous alumina samples using 120- and 200-keV STEM probes. These spectra reveal two different energy-loss mechanisms in the valence region: (1) above $10 \mathrm{eV}$ surface plasmons are excited and (2) below $10 \mathrm{eV}$, where the material is relatively transparent, the main mechanism for energy loss is the emission of Cherenkov light, which is modulated by the complex porous structure. Three different simple theoretical models have been proposed and compared with the experiments: a single pore model $(A)$, a cylindrical alumina shell $(B)$, and a triangular pore lattice $(C)$, all of them consisting of pores of infinite length. Even though the three models reproduce correctly the surface-plasmon excitation region of the spectra, they present substantial differences in the low-energy region. The origin of these differences has been explained in terms of reflection and transmission of the emitted radiation. Model $A$ allows free propagation of emitted radiation at large distances, which leads to smooth spectra. In model $B$, the emitted radiation is completely confined in the cylindrical shell by total internal reflection and the loss spectra exhibit resonances associated to light confinement. Finally, model $C$ encompasses to some extent a combination of the other two, containing both reflection in the neighboring holes and propagation beyond them. Comparison with experiment results in excellent agreement, once successive plasmon excitations have been taken into account by self-convolution of the spectra. In summary, Cherenkov radiation has been shown to be the origin of the 7-eV peak observed in the energy-loss spectra in porous alumina, and this peak comes about as a result of diffraction of this radiation in between neighboring sample holes.

\section{ACKNOWLEDGMENTS}

The authors wish to thank Gianluigi Botton (CANMET, Natural Resources Canada) and Rick Humphrey (Medical Imaging Facility of the University of Calgary) for help with the measurements and Profesor P. M. Echenique and Professor A. Howie for many enjoyable and stimulating discus- sions. Support from the Natural Sciences and Engineering Research Council (Canada), the Basque Departamento de Educación, Universidades e Investigacin, the University of the Basque Country UPV/EHU (Contract No. 00206.21513639/2001), and the Spanish Ministerio de Ciencia y Tecnologa (Contract No. MAT2001-0946) is gratefully acknowledged.

\section{APPENDIX A: COMPONENTS OF THE ELECTROMAGNETIC FIELD}

There are different ways to get the electromagnetic field, but due to the symmetry of the homogeneous solutions it can be obtained from the Hertz vectors, ${ }^{37} \vec{\Pi}=\Pi_{e} \hat{k}$ electric and $\vec{\Pi}=\Pi_{m} \hat{k}$ magnetic, respectively, directed along the $z$ axis, as follows:

$$
\begin{gathered}
\vec{E}^{H}=\boldsymbol{\nabla} \times \boldsymbol{\nabla} \times \vec{\Pi}_{e}-\frac{1}{c} \frac{\partial}{\partial t} \boldsymbol{\nabla} \vec{\Pi}_{m}, \\
\vec{H}^{H}=\frac{\epsilon_{i}}{c} \frac{\partial}{\partial t} \nabla \vec{\Pi}_{e}+\nabla \times \nabla \times \vec{\Pi}_{m} .
\end{gathered}
$$

We have assumed nonmagnetic media $\left(\mu_{i}=1\right)$. To solve the scalar wave equation for $\Pi_{e}(r, \phi, z)$ and $\Pi_{m}(r, \phi, z)$,

$$
\nabla^{2} \Psi-\frac{\epsilon_{i}}{c^{2}} \frac{\partial^{2} \Psi}{\partial t^{2}}=0
$$

we take Fourier transforms with respect to $t$ time and $z$,

$$
\Psi(r, \phi, z, t)=\frac{1}{\left(2 \pi^{2}\right)} \int_{-\infty}^{+\infty} d k \int_{-\infty}^{+\infty} d \omega \Psi_{k, \omega}(r, \phi) e^{i(k z-\omega t)}
$$

and we solve the homogeneous Bessel equations

$$
\frac{1}{r} \frac{\partial}{\partial r}\left(r \frac{\partial \Psi_{k, \omega}}{\partial r}\right)+\frac{1}{r^{2}} \frac{\partial^{2} \Psi_{k, \omega}}{\partial \phi^{2}}-\nu_{i}^{2} \Psi_{k, \omega}=0 .
$$

The general solutions of those equations are of the type

$$
\Psi_{k, \omega}(r, \phi)=\sum_{m=-\infty}^{+\infty}\left[A_{m} I_{m}\left(\nu_{i} r\right)+B_{m} K_{m}\left(\nu_{i} r\right)\right] e^{i m \phi},
$$

where the coefficients $A_{m}=0$ for region $r>b$ and $B_{m}=0$ for region $r<a$. Actually, Eq. (A5) brings a set of three different solutions, with different coefficients, for regions $r<a, a$ $<r<b$, and $r>b . I_{m}$ and $K_{m}$ are the modified Bessel functions of order $m$ and $\nu_{i}^{2}=k^{2}-\epsilon_{i}\left(\omega^{2} / c^{2}\right)$, where $\epsilon_{i}$ is the dielectric function of the corresponding medium $i=1,2,3$. To get the direct contribution to the fields, it is enough to solve the scalar wave equation with sources for the electric potential, 


$$
\begin{gathered}
\frac{1}{r} \frac{\partial}{\partial r}\left(r \frac{\partial \Phi_{k, \omega}}{\partial r}\right)+\frac{1}{r^{2}} \frac{\partial^{2} \Phi_{k, \omega}}{\partial \phi^{2}}-\nu_{i}^{2} \Phi_{k, \omega} \\
=\frac{8 \pi^{2}}{\epsilon_{i} r} \delta(r-s) \delta(\phi) \delta(\omega-k v),
\end{gathered}
$$

whose general solution is

$$
\begin{aligned}
\Phi_{k, \omega}(r, \phi)= & \frac{4 \pi}{\epsilon_{i}} \sum_{m=-\infty}^{+\infty}\left[I_{m}\left(\nu_{i} s\right) K_{m}\left(\nu_{i} r\right) \theta(r-s)\right. \\
& \left.+I_{m}\left(\nu_{i} r\right) K_{m}\left(\nu_{i} s\right) \theta(s-r)\right] \delta(\omega-k v) e^{i m \phi},
\end{aligned}
$$

where $s$ is the electron impact parameter, relative to the hole axis. The vector potential $\vec{A}=A(r, \phi, z, t) \hat{k}$ is obtained from $\Phi_{k, \omega}(r, \phi)$, using the Lorentz condition, which in the $(k, \omega)$ space means that $A_{k, \omega}(r, \phi)=\left(\epsilon_{i} \omega / c k\right) \Phi_{k, \omega}(r, \phi)$. Then the direct contribution to the fields is obtained from

$$
\vec{E}^{D}=-\nabla \Phi-\frac{1}{c} \frac{\partial \vec{A}}{\partial t}, \quad \vec{H}^{D}=\nabla \times \vec{A} .
$$

When the electron is traveling inside the pore $\left(\epsilon_{1}\right)$, the direct contribution to the electromagnetic field must be added to the homogeneous solution only in this region. For simplicity we write the electromagnetic field for the three regions, for the axial trajectory, i.e., $s=0$. Then only the terms $m=0$ remain in the expressions and $I_{0}\left(\nu_{1} s\right)=1$ :

$$
E r_{k, \omega}=\left\{\begin{array}{l}
i k \nu_{1} I_{0}^{\prime}\left(\nu_{1} r\right) a_{1}+\frac{4 \pi \nu_{1}}{\epsilon_{1}} \delta(\omega-k v) K_{0}^{\prime}\left(\nu_{1} r\right), \\
r<a \\
i k \nu_{2} I_{0}^{\prime}\left(\nu_{2} r\right) a_{2}+i k \nu_{2} K_{0}^{\prime}\left(\nu_{2} r\right) c_{2}, \quad a<r<b \\
i k \nu_{3} K_{0}^{\prime}\left(\nu_{2} r\right) a_{3}, \quad r>b,
\end{array}\right.
$$

$$
E \phi_{k, \omega}=\left\{\begin{array}{l}
-\frac{i \omega \nu_{1}}{c} I_{0}^{\prime}\left(\nu_{1} r\right) b_{1}, \quad r<a \\
-\frac{i \omega \nu_{2}}{c} I_{0}^{\prime}\left(\nu_{2} r\right) b_{2}-\frac{i \omega \nu_{2}}{c} K_{0}^{\prime}\left(\nu_{2} r\right) d_{2}, \\
a<r<b \\
-\frac{i \omega \nu_{3}}{c} K_{0}^{\prime}\left(\nu_{3} r\right) b_{3}, \quad r>b,
\end{array}\right.
$$

$$
\begin{aligned}
& E z_{k, \omega}= \begin{cases}-\nu_{1}^{2} I_{0}\left(\nu_{1} r\right) a_{1}+i \frac{4 \pi}{\epsilon_{1}} \delta(\omega-k v)\left(k-\frac{v \epsilon_{1} \omega}{c^{2}}\right) K_{0}\left(\nu_{1} r\right), & r<a \\
-\nu_{2}^{2} I_{0}\left(\nu_{2} r\right) a_{2}-\nu_{2}^{2} K_{0}\left(\nu_{2} r\right) c_{2}, & a<r<b \\
-\nu_{3}^{2} K_{0}\left(\nu_{3} r\right) a_{3}, & r>b,\end{cases} \\
& H r_{k, \omega}= \begin{cases}i k \nu_{1} I_{0}^{\prime}\left(\nu_{1} r\right) b_{1}, & r<a \\
i k \nu_{2} I_{0}^{\prime}\left(\nu_{2} r\right) b_{2}+i k \nu_{2} K_{0}^{\prime}\left(\nu_{2} r\right) d_{2}, & a<r<b \\
i k \nu_{3} K_{0}^{\prime}\left(\nu_{3} r\right) b_{3}, & r>b,\end{cases} \\
& H \phi_{k, \omega}= \begin{cases}\frac{i \omega \nu_{1}}{c} \epsilon_{1} I_{0}^{\prime}\left(\nu_{1} r\right) a_{1}+\frac{4 \pi v}{c} \epsilon_{1} \nu_{1} \delta(\omega-k v) K_{0}^{\prime}\left(\nu_{1} r\right), & r<a \\
\frac{i \omega \nu_{2}}{c} \epsilon_{2} I_{0}^{\prime}\left(\nu_{2} r\right) b_{2}-\frac{i \omega \nu_{2}}{c} K_{0}^{\prime}\left(\nu_{2} r\right) c_{2}, & a<r<b \\
\frac{i k \omega \nu_{3} v}{c} \nu_{3} \epsilon_{3} K_{0}^{\prime}\left(\nu_{3} r\right) a_{3}, & r>b,\end{cases} \\
& H z_{k, \omega}= \begin{cases}-\nu_{1}^{2} I_{0}\left(\nu_{1} r\right) b_{1}, & r<a \\
-\nu_{2}^{2} I_{0}\left(\nu_{2} r\right) b_{2}-\nu_{2}^{2} K_{0}\left(\nu_{2} r\right) d_{2}, & a<r<b \\
-\nu_{3}^{2} K_{0}\left(\nu_{3} r\right) b_{3}, & r>b .\end{cases}
\end{aligned}
$$


By matching the boundary conditions, i.e., continuity of $\epsilon_{i} E r, E \phi, E z$, and $\vec{H}$ at the cylindrical interfaces $r=a$ and $r=b$, we get a system of eight independent equations with eight coefficients. Then the energy loss experienced by the moving electron is obtained from the induced electric field on the electron in the $z$ direction. The system is solved for the $a_{1}$ coefficient, using the MATHEMATICA package and finally we get

$$
a_{1}=\frac{i}{k} \frac{4 \pi}{\epsilon_{1}} \delta(\omega-k v) I_{0}\left(\nu_{1} s\right) F(\omega),
$$

where the function $F(\omega)$ has been written in Sec. III. With that coefficient we can obtain $E z_{k, \omega}$ and from it, the energy lost by the swift beam electron moving along the cylindrical cavity.

\section{APPENDIX B: CHERENKOV MODES IN A CYLINDRICAL SHELL}

In the energy-loss probability given by the formula (4), the Bessel functions have complex arguments. Let us consider an electron moving along the axis of a cylindrical shell of a medium of dielectric constant $\epsilon$, i.e., there is vacuum outside of the shell $\left(\epsilon_{1}=\epsilon_{3}=1\right)$. Now, the expression for the energy-loss probability per unit length can be written as the ratio of two real functions, as follows:

$$
\frac{d P(\omega)}{d z}=\frac{2 \gamma}{\pi v^{2}} \operatorname{Im}\left\{\frac{\Gamma(\omega)}{\Delta(\omega)}\right\}
$$

where $\gamma=\sqrt{1-\beta^{2}}$ and functions $\Gamma(\omega)$ and $\Delta(\omega)$ are real and defined as follows:

$$
\begin{aligned}
\Gamma(\omega)= & \nu_{1}^{2} \epsilon^{2} K_{0}\left(\nu_{1} a\right) K_{0}\left(\nu_{1} b\right) \Xi_{1}(\omega) \\
& -\nu_{2}^{2} K_{1}\left(\nu_{1} a\right) K_{1}\left(\nu_{1} b\right) \Xi_{2}(\omega) \\
& +\nu_{1} \nu_{2} \epsilon\left\{K_{0}\left(\nu_{1} a\right) K_{1}\left(\nu_{1} b\right) \Xi_{3}(\omega)\right. \\
& \left.-K_{1}\left(\nu_{1} a\right) K_{0}\left(\nu_{1} b\right) \Xi_{4}(\omega)\right\}, \\
\Delta(\omega)= & \nu_{1}^{2} \epsilon^{2} I_{0}\left(\nu_{1} a\right) K_{0}\left(\nu_{1} b\right) \Xi_{1}(\omega) \\
& +\nu_{2}^{2} I_{1}\left(\nu_{1} a\right) K_{1}\left(\nu_{1} b\right) \Xi_{2}(\omega) \\
& +\nu_{1} \nu_{2} \epsilon\left\{I_{0}\left(\nu_{1} a\right) K_{1}\left(\nu_{1} b\right) \Xi_{3}(\omega)\right. \\
& \left.+I_{1}\left(\nu_{1} a\right) K_{0}\left(\nu_{1} b\right) \Xi_{4}(\omega)\right\},
\end{aligned}
$$

where $K_{1}$ and $I_{1}$ stand for the modified Bessel functions of order 1 , and $\nu_{1}=(\omega / v) \gamma$ and $\nu_{2}=(\omega / v) \sqrt{\epsilon \beta^{2}-1}$ in this particular case. $\Xi_{i}(\omega), i=1,2,3,4$ are real functions defined as follows:

$$
\begin{aligned}
\Xi_{1}= & J_{1}\left(\nu_{2} a\right) J_{1}\left(\nu_{2} b\right) \ln \left(\frac{b}{a}\right)-J_{1}\left(\nu_{2} a\right) \xi_{1}\left(\nu_{2} b\right) \\
& +J_{1}\left(\nu_{2} b\right) \xi_{1}\left(\nu_{2} a\right)
\end{aligned}
$$

$$
\begin{aligned}
\Xi_{2}= & -J_{0}\left(\nu_{2} a\right) J_{0}\left(\nu_{2} b\right) \ln \left(\frac{b}{a}\right)+J_{0}\left(\nu_{2} a\right) \xi_{0}\left(\nu_{2} b\right) \\
- & J_{0}\left(\nu_{2} b\right) \xi_{0}\left(\nu_{2} a\right), \\
\Xi_{3}= & J_{0}\left(\nu_{2} b\right) J_{1}\left(\nu_{2} a\right)\left\{\ln \left(\frac{b}{a}\right)+\gamma\right\} \\
& +J_{0}\left(\nu_{2} b\right) \xi_{1}\left(\nu_{2} a\right)-J_{1}\left(\nu_{2} a\right) \xi_{0}\left(\nu_{2} b\right), \\
\Xi_{4}= & -J_{0}\left(\nu_{2} a\right) J_{1}\left(\nu_{2} b\right)\left\{\ln \left(\frac{b}{a}\right)-\gamma\right\} \\
& +J_{0}\left(\nu_{2} a\right) \xi_{1}\left(\nu_{2} b\right)-J_{1}\left(\nu_{2} b\right) \xi_{0}\left(\nu_{2} a\right),
\end{aligned}
$$

where $J_{0,1}$ are the Bessel functions of integer order. The constant $\gamma$ present in the definitions of functions $\Xi_{3}$ and $\Xi_{4}$ is Euler's constant. $\xi_{0,1}$ are real functions defined as follows:

$$
\begin{gathered}
\xi_{0}(x)=\sum_{k=1}(-1)^{k}\left\{1+\frac{1}{2}+\frac{1}{3}+\cdots+\frac{1}{k}\right\} \frac{\left(\frac{x}{2}\right)^{2 k}}{(k !)^{2}}, \\
\xi_{1}(x)=\frac{1}{x}+\frac{x}{4} \sum_{k=0}(-1)^{k}\{\psi(k+1)+\psi(k+2)\} \frac{\left(\frac{x}{2}\right)^{2 k}}{k !(k+1) !},
\end{gathered}
$$

where $\psi(k)$ stands for the digamma function (see Ref. 38). In Eq. (B1) the only contribution to the energy-loss probability spectrum arises from the zeros of the $\Delta$, and can be written as follows:

$$
\frac{d P(\omega)}{d z}=\frac{2 \gamma}{v^{2}} \sum_{i} \frac{\Gamma(\omega)}{\frac{d \Delta(\omega)}{d \omega}} \delta\left(\omega-\omega_{i}\right),
$$

where $\omega_{i}$ are zeros of the function $\Delta$. Then, we get peaks in the EELS spectra, centered at the energies corresponding to the zeros of $\Delta(\omega)$.

Assuming that the penetration of the radiation into both media 1 and 3 is much smaller than $a$, the equation of the modes of the shell, $\Delta(\omega)=0$, can be recast as

$$
J_{0}\left(\nu_{2} a\right) Y_{0}\left(\nu_{2} b\right)-J_{0}\left(\nu_{2} b\right) Y_{0}\left(\nu_{2} a\right)=0,
$$

where $Y_{0}(x)$ stands for the Bessel function of integer order. The $n$th zero of this equation, $\nu_{2}^{(n)}$, can be written as a series expansion: ${ }^{38}$

$$
\nu_{2}^{(n)}=n \frac{\pi}{b-a}\left\{1-\frac{1}{8 n^{2} \pi^{2}} \frac{(b-a)^{2}}{a b}+\cdots\right\} .
$$

The first term in Eq. (B6) is similar to the condition for standing waves in a well. 
${ }^{1}$ L. Reimer, Mater. Trans., JIM 39, 873 (1998).

${ }^{2}$ W. Grogger, F. Hofer, P. Warbichler, and G. Kothleitner, Microsc. Microanal. 6, 161 (2000).

${ }^{3}$ R.F. Egerton, Electron Energy-loss Spectroscopy in the Electron Microscope (Plenum Press, New York, 1996).

${ }^{4}$ C.V. Festenberg, Z. Phys. 207, 47 (1969).

${ }^{5}$ A. Rivacoba, N. Zabala, and J. Aizpurua, Prog. Surf. Sci. 65, 1 (2000).

${ }^{6}$ R.H. Milne and P.M. Echenique, Solid State Commun. 55, 909 (1985).

${ }^{7}$ P. Moreau, N. Brun, C.A. Walsh, C. Colliex, and A. Howie, Phys. Rev. B 56, 6774 (1997).

${ }^{8}$ V.L. Ginzburg, Phys. Usp. 39, 973 (1996).

${ }^{9}$ R. García-Molina, A. Gras-Martí, A. Howie, and R.H. Ritchie, J. Phys. C 18, 5335 (1985).

${ }^{10}$ F.J. García de Abajo, A. Rivacoba, N. Zabala, and N. Yamamoto, Phys. Rev. B (to be published).

${ }^{11}$ J. Daniels, C.v. Festenberg, H. Raether, and K. Zeppenfeld, Springer Tracts Mod. Phys. 54, 78 (1970).

${ }^{12}$ C.H. Chen, J. Silcox, and R. Vincent, Phys. Rev. B 12, 64 (1975).

${ }^{13}$ N. Yamamoto, K. Araya, A. Toda, and H. Sugiyama, Surf. Interface Anal. 31, 79 (2001); N. Yamamoto, K. Araya, and F.J. Garcia de Abajo, Phys. Rev. B 64, 205419 (2001).

${ }^{14}$ H. Abe, H. Kurata, and K. Hojou, J. Phys. Soc. Jpn. 69, 1553 (2000).

${ }^{15}$ P. Williams, C. Levy-Clement, A. Albu-Yaron, N. Brun, and C. Colliex, J. Porous Mater. 7, 159 (2000).

${ }^{16}$ C.R. Martin, Chem. Mater. 8, 1739 (1996).

${ }^{17}$ A.P. Li, F. Müller, A. Birner, K. Nielsch, and U. Gösele, Adv. Mater. 11, 483 (1999).

${ }^{18}$ C.R. Martin, Science 266, 1961 (1994).

${ }^{19}$ G. Yi and W. Schwarzacher, Appl. Phys. Lett. 74, 1746 (1999).

${ }^{20}$ R.E. Benfield, D. Grandjean, J.C. Dore, Z. Wu, M. Kröll, T. Sawitowski, and G. Schmid, Eur. Phys. J. D 16, 399 (2001).
${ }^{21}$ D. Arayasantiparb, S. McKnight, and M. Libera, J. Adhes. 76, 353 (2001).

${ }^{22}$ F.J. García de Abajo and A. Howie, Phys. Rev. Lett. 80, 5180 (1998); Phys. Rev. B 65, 115418 (2002).

${ }^{23}$ H. Masuda and K. Fukada, Science 268, 1466 (1995).

${ }^{24}$ D. Crouse, Y.H. Lo, A.E. Miller, and M. Crouse, Appl. Phys. Lett. 76, 49 (2000).

${ }^{25}$ E.D. Palik, Handbook of Optical Constants of Solids (Academic, London, 1985).

${ }^{26}$ C.A. Walsh, Philos. Mag. A 59, 227 (1989).

${ }^{27}$ N. Zabala, A. Rivacoba, and P.M. Echenique, Surf. Sci. 209, 465 (1989).

${ }^{28}$ M.E. Mochel, C.J. Humphreys, J.A. Eades, J.M. Mochel, and A.K. Petford, Appl. Phys. Lett. 42, 392 (1983); M. Scheinfein, A. Murray, and M. Isaacson, Ultramicroscopy 16, 233 (1985); J.M. Macaulay, R.M. Allen, L.M. Brown, and S.D. Berger, Microelectron. Eng. 9, 557 (1989).

${ }^{29}$ P.M. Echenique and J.B. Pendry, J. Phys. C 8, 2936 (1975).

${ }^{30}$ N. Zabala, A. Rivacoba, F.J. García de Abajo, and A. Pattantyus, Surf. Sci. 532, 461 (2003); F.J. Garcia de Abajo, A.G. Pattantyus-Abraham, N. Zabala, A. Rivacoba, M.O. Wolf, and P.M. Echenique, Phys. Rev. Lett. 91, 143902 (2003).

${ }^{31}$ J. D. Jackson, Classical Electrodynamics (Wiley, New York, 1975).

${ }^{32}$ W. Lu and J. Dong, Phys. Rev. B 63, 033401 (2001).

${ }^{33}$ H.A. Olsen and H. Kolbenstvedt, Phys. Rev. A 21, 1987 (1980).

${ }^{34}$ J. Osma and F.J. García de Abajo, Phys. Rev. A 56, 2032 (1997).

${ }^{35}$ R.H. French, H. Müllejans, and D.J. Jones, J. Am. Ceram. Soc. 81, 2549 (1998).

${ }^{36}$ J. Aizpurua, A. Howie, and F.J. García de Abajo, Phys. Rev. B 60, 11149 (1999).

${ }^{37}$ J. A. Stratton, Electromagnetic Theory (McGraw-Hill, New York, 1941).

${ }^{38}$ A. Abramowitz and I. Stegun, Handbook of Mathematical Functions (Dover, New York, 1965). 